\title{
Management of peripheral arterial disease in the elderly: focus on cilostazol
}

\author{
Travis M Falconer' \\ John W Eikelboom² \\ Graeme J Hankey ${ }^{3}$ \\ Paul E Norman' \\ 'School of Surgery, University of \\ Western Australia, Fremantle Hospital, \\ Western Australia; ${ }^{2}$ Department \\ of Medicine, McMaster University, \\ Hamilton, Canada; ${ }^{3}$ Department of \\ Neurology, Royal Perth Hospital, \\ School of Medicine and Pharmacology, \\ University of Western Australia
}

Correspondence: Professor Paul E Norman

School of Surgery, University of Western Australia, Fremantle Hospital, PO Box 480, Fremantle 6959,WA, Australia

Tel +6 I 8 943। 2500

Fax +61894312623

Email paul.norman@uwa.edu.au

\begin{abstract}
Symptomatic and asymptomatic peripheral arterial disease (PAD) is a common problem in the elderly. The management of PAD includes the prevention of cardiovascular events and relief of symptoms - most commonly intermittent claudication (IC). Both require treatment of the causes and consequences of atherothrombosis, but some strategies are more effective for prevention of cardiovascular events and others are more effective for the relief of symptoms. Priorities for the prevention of cardiovascular events include smoking cessation, exercise, antiplatelet therapy, and the treatment of dyslipidemia, hypertension, and diabetes. Walking time and ability are improved by exercise. The benefit of numerous drugs in the treatment of IC has been assessed. The results have generally been disappointing, but there is some evidence that statins and cilostazol (an inhibitor of phosphodiesterase 3) are of benefit. Meta-analyses suggest that cilostazol increases maximum walking distance by $40 \%-50 \%$ and improves other objective measures of walking. The safety profile of cilostazol in patients with PAD appears to be acceptable although the mechanism for its effect on IC is unclear. In addition to risk factor management, treatment with cilostazol should be considered in patients with disabling IC.
\end{abstract}

Keywords: peripheral arterial disease, intermittent claudication, risk factors, cilostazol

Peripheral arterial disease (PAD) refers to the pathological process of obstruction of the arteries excluding those of the coronary and cerebral circulation. It most commonly affects the arteries of the lower limbs and is caused by atherothrombosis or other vascular disease including embolism, arteritis, and aneurysm. The management of PAD has historically focused on preventing local complications such as critical limb ischemia and limb loss. More recently there has been a greater emphasis on preventing systemic complications of the disease and improving the management of disabling symptoms, specifically intermittent claudication (IC). This article will review the medical management of PAD in the elderly, with a particular focus on the role of cilostazol in treating the symptoms of IC. The management of severe disease causing rest pain or tissue loss and needing re-vascularization or amputation will not be reviewed.

\section{Diagnosis of peripheral arterial disease}

Intermittent claudication is defined as the painful aching, cramping, or tightness of muscles during exercise, due to insufficient blood flow to meet the metabolic demands. It usually presents in the calf when the infra-inguinal arteries are affected, and may also present in the buttocks and thighs if there is aorto-iliac disease. Some patients with PAD, particularly the elderly, may not experience IC because of co-morbidities that limit walking such as arthritis, spinal stenosis, heart failure, and pulmonary disease (McDermott et al 2001). Consequently, a more sensitive diagnostic test for PAD is required for the identification of patients with asymptomatic PAD: the Ankle Brachial Pressure Index (ABPI). 
The ABPI is defined as the ratio of the highest systolic pressure at the ankle divided by the brachial systolic pressure (Naylor et al 1988). It has been widely accepted that an ABPI of less than 0.9 is up to $95 \%$ sensitive and $99 \%$ specific in detecting peripheral arterial disease that would be detected on angiography (Dormandy and Rutherford 2000; Aronow 2004). This non-invasive bedside test is a reliable tool in assessing the presence and severity of PAD rather than relying solely on a clinical diagnosis. An ABPI of less than 0.9 is associated with an increased risk of cardiovascular and cerebrovascular events (Newman et al 1999). The benefit of early diagnosis by screening for asymptomatic PAD (defined by a ABPI of $<0.9$ ), is debated but remains to be established (Beckman et al 2006).

\section{Prevalence of peripheral arterial disease}

The reported prevalence of PAD depends on the diagnostic criteria used to define PAD (Hiatt et al 1995). The prevalence of symptomatic and asymptomatic PAD increases markedly with age, smoking, and diabetes (Selvin and Erlinger 2004). There are weaker associations with male gender, non-white ethnicity, hypertension, dyslipidemia, and impaired renal function (Norgren et al 2007). The prevalence of IC increases from about $2 \%$ in those aged $50-54$ years to $7 \%$ in those aged 70-74 years (Norgren et al 2007). However, only about one third of patients with PAD (defined as an ABPI $<0.9$ ) are symptomatic with IC (Selvin and Erlinger 2004; Norgren et al 2007).

\section{Medical management of peripheral arterial disease}

The twin priorities in the medical management of PAD are symptom relief and the prevention of cardiovascular events. Both require treatment of the causes and consequences of atherothrombosis but some strategies are more effective for one goal than the other.

\section{Smoking cessation}

The association between smoking and PAD is very strong with over $80 \%$ of patients being current or ex-smokers (Norgren et al 2007). Furthermore, the association is dosedependent; increasing exposure to cigarette smoke is associated with earlier onset of symptoms and more severe PAD (Fowler et al 2002; Willgendael et al 2004). Finally, smoking cessation in patients with $\mathrm{PAD}$ is associated with a reduction in severity of IC and risk of developing rest pain (Girolami et al 1999), albeit to a lesser degree than the reduction in risk of coronary heart disease and stroke (Willgendael et al 2004). This may drive some of the increasing incidence seen in the elderly. Rates of smoking cessation are improved by such methods as physician advice, nicotine replacement, and bupropion therapies (Hankey et al 2006).

\section{Exercise and weight loss}

Regular exercise is not only beneficial for cardiovascular health among the general population (Shepard and Balady 1999) but also for patients with PAD in whom it reduces mortality and cardiovascular events (Garg et al 2006). Exercise is also beneficial in improving maximum walking time and walking ability (Wind and Koelemay 2007). However, it remains to be established whether structured or supervised exercise programs are better than unsupervised exercise (Fowler et al 2002; Wind and Koelemay 2007). Obesity is associated with increased cardiovascular risk (Yusef et al 2005), but the effectiveness of weight loss in reducing risk and improving IC remains to be established.

\section{Dyslipidemia}

Epidemiological studies suggest that dyslipidemia is a modest risk factor for PAD (Selvin and Erlinger 2004; Norgren et al 2007). However, among 2701 patients with a history of PAD but no prior coronary heart disease, lowering LDL cholesterol by about $1.0 \mathrm{mmol} / \mathrm{L}$ by means of simvastatin treatment $(40 \mathrm{mg} / \mathrm{d})$ for 5 years resulted in a significant $19 \%$ reduction in the relative risk of cardiovascular events compared with placebo (Heart Protection Study Collaborative Group 2002). Random assignment to simvastatin has also been associated with a significant reduction in the incidence of new IC (relative risk 0.62, 95\% CI: 0.44-0.88) (Pedersen et al 1998) and an improvement in several objective measures of walking among those who experience IC (Erez and Leitersdorf 2007).

\section{Treating hypertension}

Hypertension has been reported to be a risk factor for PAD in some but not all studies (Selvin and Erlinger 2004; Norgren et al 2007). Although there are few data based on patients with PAD, drug treatment of hypertension reduces cardiovascular risk (Blood pressure lowering treatment trialists' collaboration 2003; Mehler et al 2003). The HOPE study and other large trials have established that angiotensin-converting enzyme (ACE) inhibitors significantly reduce the risk of cardiovascular related events in patients with symptomatic and asymptomatic PAD (Hankey et al 2006; Heart Outcome Prevention Evaluation Study Investigators 2000). In addition 
to the reduction in cardiovascular risk, ACE inhibitors may also improve walking distances (Hankey et al 2006).

\section{Treating diabetes}

Intermittent claudication is almost twice as common in patients with diabetes compared to those with normal glycemic control (Norgren et al 2007). Even in the absence of diabetes, insulin resistance increases the risk of PAD by nearly 50\% (Muntner et al 2005). Intensive glycemic control reduces the impact of microvascular complications of diabetes, but there is little evidence that it changes the course of macrovascular disease causing PAD (Adler et al 2002). Neuropathy, local microvascular changes in the foot and PAD all contribute to diabetic foot problems and screening for these has been advocated (American Diabetes Association 2003).

\section{Antiplatelet therapy}

Antiplatelet therapy (chiefly aspirin) reduces the relative risk of major cardiovascular events among those at risk of atherothrombosis, including those with IC, by about $25 \%$ (Antithrombotic Trialists' Collaboration 2002). Among 19,000 individuals at high vascular risk randomly assigned to long-term aspirin or clopidogrel in the CAPRIE trial, a subgroup analysis of the 6000 patients with PAD showed that those assigned clopidogrel experienced a $23.8 \%(95 \%$ CI: $9 \%-36 \%$ ) reduction in the relative risk of major cardiovascular events compared to aspirin (CAPRIE Steering Committee 1996). There is no evidence that aspirin or clopidogrel improve IC.

\section{Role of cilostazol in the treatment of intermittent claudication}

Over the past three decades there have been numerous trials to assess the influence of various drugs on walking distances and the severity of IC. The majority of these drugs have been disappointing and the results of the trials are reviewed elsewhere (Hiatt 2001; Aronow 2006). An exception is cilostazol, which has emerged over the past decade as a safe and effective drug in the management of IC (Thompson et al 2002).

\section{Mechanism of action}

Cilostazol has antiplatelet and vasodilatory effects which are believed to be mediated by inhibition of phosphodiesterase 3 (PDE3) which results in increased concentrations of intracellular cAMP (Bradbury 2003). Cilostazol inhibits primary and secondary platelet aggregation, and high-shear stress platelet aggregation both in vivo and in vitro (Reilly and Mohler 2001). Shear stress induced during exercise is thought to be an important mechanism of platelet activation in patients with atherothrombosis and is more effectively inhibited by cilostazol than aspirin (Reilly and Mohler 2001). Cilostazol also increases smooth muscle cell cAMP, resulting in a vasodilatory effect which may benefit patients with IC (Takahashi et al 1992; Matousovic et al 1995; Liu et al 2001).

Cilostazol also reduces plasma triglycerides and increases plasma HDL concentrations (Elam et al 1998; Beebe et al 1999; Dawson et al 2000). The mechanism is unclear but it is thought to be related to increased levels of cAMP, increased lipoprotein lipase activity, and attenuated triglyceride secretion (Motoyashiki et al 1996; Elam et al 1998).

Cilostazol has no effect on the prothrombotic, hypofibrinolytic state that may develops in patients with IC (Hobbs et al 2007).

Despite these insights, the mechanism of action of cilostazol in IC remains uncertain.

\section{The efficacy of cilostazol in intermittent claudication}

There have been a number of trials examining the influence of cilostazol on maximal walking distances (MWD), pain free walking distance (PFWD), patient's perception of improvement, and plasma lipids. A meta-analysis (Thompson et al 2002) identified 8 phase III randomized, double blind, placebo-controlled trials that involved a total of 2702 patients with stable, moderate to severe claudication (Table 1). Inclusion criteria were: aged 40 years or older with symptomatic PAD for $>6$ months and no significant change in severity for a minimum of 3 months. Exclusion criteria were: rest pain, Buerger's disease, ischemic tissue necrosis, surgical or endovascular procedures within the past 3 months, unstable coronary artery disease, symptomatic cardiac arrhythmias, recent ( $<3$ months) DVT, conditions that limited exercise capacity other than IC; and usage of $>81 \mathrm{mg} /$ day of aspirin or $1200 \mathrm{mg} /$ day ibuprofen. Intermittent claudication was defined uniformly across all studies as "cramping, aching, fatigue, or any patient-reported pain in one or both legs that occurred during walking that was sufficient to cause the subject to reduce the pace or cease ambulation". An ABPI of $<0.9$ ( 7 trials) or $<0.8$ ( 1 trial) was used to diagnose PAD and this was confirmed by a decrease of $>10 \mathrm{mmHg}$ in ankle systolic pressure post exercise. All studies also included patients with a decrease in ankle systolic pressure greater than $20 \mathrm{mmHg}$ post exercise even if they did not fit 
Table I Randomized controlled trials of cilostazol in patients with PAD including those used in the meta-analysis by Thompson et al (2002)

\begin{tabular}{|c|c|c|c|c|}
\hline Study & Treatment groups & Participants & Duration (weeks) & Treadmill design \\
\hline \multirow[t]{3}{*}{ Beebe et al 1999} & Cilostazol $50 \mathrm{mg}$ bd & 171 & 24 & Constant load \\
\hline & Cilostazol $100 \mathrm{mg}$ bd & 175 & & \\
\hline & Placebo & 170 & & \\
\hline \multirow[t]{2}{*}{ Dawson et al 1998} & Cilostazol $100 \mathrm{mg}$ bd & 54 & 12 & Constant load \\
\hline & Placebo & 27 & & \\
\hline \multirow[t]{3}{*}{ Dawson et al 2000} & Cilostazol 100 mg bd & 227 & 24 & Variable load \\
\hline & Pentoxifylline $400 \mathrm{mg}$ tds & 232 & & \\
\hline & Placebo & 239 & & \\
\hline \multirow[t]{2}{*}{ Elam et al 1998} & Cilostazol $100 \mathrm{mg}$ bd & 95 & 12 & Variable load \\
\hline & Placebo & 94 & & \\
\hline \multirow[t]{4}{*}{ Hobbs et al 2007} & Best Medical Therapy (BMT) & 9 & 24 & Constant load \\
\hline & BMT + Cilostazol & 9 & & \\
\hline & BMT + Cilostazol + Supervised exercise & 7 & & \\
\hline & BMT + Supervised exercise & 9 & & \\
\hline \multirow[t]{2}{*}{ Money et al 1998} & Cilostazol 100 mg bd & 119 & 16 & Variable load \\
\hline & Placebo & 120 & & \\
\hline \multirow[t]{3}{*}{ Otsuka 1996a } & Cilostazol $100 \mathrm{mg}$ bd & 72 & 12 & Constant load \\
\hline & Cilostazol $150 \mathrm{mg}$ bd & 73 & & \\
\hline & Placebo & 70 & & \\
\hline \multirow[t]{3}{*}{ Otsuka 1996b } & Cilostazol 100 mg bd & 123 & 24 & Constant load \\
\hline & Pentoxifylline $400 \mathrm{mg}$ tds & 123 & & \\
\hline & Placebo & 124 & & \\
\hline \multirow[t]{3}{*}{ Strandness et al 2002} & Cilostazol 50 mg bd & 132 & 24 & Constant load \\
\hline & Cilostazol $100 \mathrm{mg}$ bd & 133 & & \\
\hline & Placebo & 129 & & \\
\hline
\end{tabular}

Key: bd, twice daily; tds, three times daily.

the ABPI index definition. These patients were then required to reproduce exercise ability on 2-3 standardized treadmill walking tests with less than $20 \%$ variation.

Participants in all studies were assessed with monthly medical history, physical examination, blood testing, treadmill testing, Doppler measurements and administration of standardised questionaries assessing functional status and quality of life. Treadmill tests were either standardised progressive workload or constant workload formats. All of the trials used the percentage change in MWD as their primary outcome and the change in PFWD as a secondary outcome. Quality of life was assessed using the Medical Outcomes Scale Form Health Survey 36 (SF-36) and the Walking Impairment Questionnaire (WIQ). The WIQ examined walking speed, distance and degree of impairment and was used in 6 of the 8 studies.

After exclusion of patients receiving the $150 \mathrm{mg}$ dosing (used in one trial only), data from 2399 patients were analyzed (Thompson et al 2002). The mean age (years, \pm SD) of the cilostazol and placebo groups was $65( \pm 9.24)$ and $66( \pm 8.78)$ respectively. After treatment for 12-24 weeks, there was a statistically significant increase in MWD for patients receiving cilostazol $50 \mathrm{mg}$ bd or $100 \mathrm{mg}$ bd compared with placebo (increases of $44 \%, 50 \%$, and $21 \%$ respectively). Significant changes in PFWD were seen in 5 of the 8 trials although there were no differences between subgroups based on age, gender, or diabetes. It should be noted that the percentage increases in MWD and PFWD for the placebo groups highlights the large placebo effect in these studies.

The two trials including pentoxifylline showed no statistical difference from placebo. After 24 weeks, there was a $12.8 \%$ increase in high-density lipoprotein cholesterol $(\mathrm{p}=0.0001)$ and a $15.8 \%$ decrease in triglycerides ( $p=0.0001$ ) in patients receiving the $100 \mathrm{mg}$ dose compared with placebo and pentoxifylline. There were no significant differences in hematological markers, liver function tests, renal function, or electrolytes between all three groups (Thompson et al 2002).

Regensteiner et al (2002) performed another metaanalysis on 6 of the 8 studies included in that by Thompson et al focusing on the effects of cilostazol (100 $\mathrm{mg} \mathrm{bd})$ on community based walking ability and quality of life measures. They believed that treadmill walking did not appropriately reflect patients' perceived ability to walk in the community and the effect on quality of life. The 6 studies 
included all used the SF-36 and the WIQ and the patients receiving a dose of $150 \mathrm{mg}$ of cilostazol or pentoxifylline were excluded, bringing the total number to 1751 (Regensteiner et al 2002). The mean age (years \pm SD) of the patients analysed in both the cilostazol and placebo groups in this meta-analysis was $65( \pm 9)$. The WIQ scores were significantly better in patients taking cilostazol compared with placebo. The physical summary score on the SF-36 improved $5 \%$ in those patients taking cilostazol along with increases in the subscale scores of physical functioning, limitations/problems, and bodily pain. However, patients taking placebo also improved their physical function score significantly ( $p<0.05$ ), albeit to a lesser degree than that seen with cilostazol. It was concluded that the WIQ and the SF-36 appropriately reflected increases in MWD and PFWD through examining patients' perceived ability to walk in a community based setting and their health related quality of life (Regensteiner et al 2002).

Further evidence of the efficacy of cilostazol was demonstrated in a study involving 59 patients randomized to cilostazol or pentoxifylline and then crossed over to placebo for 6 weeks of treatment. Patients receiving cilostazol lost $49 \%$ of the increased MWD after 24 weeks of treatment when the drug was stopped, an effect not seen with pentoxifylline (Dawson 2001).

Three randomized controlled trials involving cilostazol examined its effect on ABPI with mixed results. Money et al (1998), whose study involved 239 patients found that was a marginal increase in ABPI in the cilostazol group $(0.64 \pm 0.02$ to $0.7 \pm 0.02)$ compared with the placebo group $(0.68 \pm 0.02$ to $0.69 \pm 0.02$ ). Other studies also showed extremely modest improvements in ABPI, without any statistical significance (Dawson et al 1998; Hobbs et al 2007).

\section{Dose response relationship}

Beebe et al 1999 analyzed the data from 516 patients randomized to 1 of 3 groups: cilostazol $50 \mathrm{mg}$ twice daily, cilostazol $100 \mathrm{mg}$ twice daily, or placebo (Beebe et al 1999). Patients in this trail were tested on a constant grade treadmill with a grade of $12.5 \%$ and at a speed of $3.2 \mathrm{~km} / \mathrm{h}$. Patients receiving $50 \mathrm{mg}$ twice daily increased their walking distances by almost 1.5 fold (MWD from $132 \mathrm{~m}$ to $199 \mathrm{~m}$ and PFWD from $67 \mathrm{~m}$ to $115 \mathrm{~m}$ at 24 weeks). This increase was proportionately larger in those receiving cilostazol $100 \mathrm{mg}$ twice daily with an almost 2-fold increase (MWD from $130 \mathrm{~m}$ to $259 \mathrm{~m}$ and PFWD from $70 \mathrm{~m}$ to $138 \mathrm{~m}$ at 24 weeks) (Beebe et al 1999). Using extrapolations based on energy expenditure, it has been estimated that patients taking cilostazol $50 \mathrm{mg}$ twice daily would double their walking distances and those receiving cilostazol $100 \mathrm{mg}$ twice daily would triple their maximal walking distances (Cariski 2001).

\section{Use in diabetic patients}

The efficacy of cilostazol (100 mg twice daily) in diabetic patients has been reviewed by Rendell et al (2002). Using data from 8 trials, 216 diabetic patients and 599 non-diabetic patients receiving cilostazol were compared with 220 diabetic and 616 non-diabetic patients receiving placebo. There was no significant difference in increased percentage of MWD (51\% vs 61\%) between the diabetic and non-diabetic patients treated with cilostazol. Among patients with more severe claudication (shortest baseline MWD) those with diabetes experienced a greater percentage increase in MWD with cilostazol than those without diabetes (34\% change from baseline MWD in Q1 compared with 5.5\%, 23\% and 17.2\% in Q2, Q3, Q4, log transformed data) (Rendell et al 2002).

\section{Safety and side effect profile}

Pratt reviewed the safety data from the 8 randomized control trials in an analysis that included 1374 patients receiving cilostazol for 12-24 weeks (475 patient exposure years) (Pratt 2001). The most common side effects associated with cilostazol were headache, diarrhoea, and other gastrointestinal complaints; $1.3 \%$ of patients on cilostazol $50 \mathrm{mg}$ twice daily and $3.7 \%$ on $100 \mathrm{mg}$ twice daily had to discontinue the drug due to headache compared with only $0.3 \%$ of patients on placebo. The rate of cardiovascular events including myocardial infarction and stroke were similar in all three of the groups (Pratt 2001). Exacerbation of pre-existing cardiac failure in was similar (5.5\% vs $4.8 \%$ ) with cilosta$\mathrm{zol}(\mathrm{n}=55)$ and placebo $(\mathrm{n}=42)$. Very few patients treated with cilostazol $(7 / 973,0.72 \%)$ developed new onset heart failure compared with those on placebo $(12 / 1374,0.87 \%)$ (Pratt 2001). However, cilostazol remains contraindicated in patients with heart failure.

\section{Conclusion}

The management of PAD and IC are important issues for all clinicians treating the aging population. Early diagnosis and optimal medical management has the potential to provide patients with the best quality and longevity of life. The emergence of cilostazol as a pharmacological treatment of IC has added a further option to the clinician's armamentarium in managing these patients. Cilostazol increases maximal walking distance, pain free walking distance, and quality of 
life and is superior to other medical options for treatment of IC. It is contraindicated in patients with heart failure but is safe in the elderly and diabetic patients and has a tolerable side effect profile. Cilostazol is available in $50 \mathrm{mg}$ and $100 \mathrm{mg}$ preparations in both the US and UK markets.

\section{Disclosures}

Dr Eikelboom has received honoraria and/or research funding from companies that market antithrombotic drugs (Bayer, BMS, GSK, McNeil, Pfizer, Sanofi-Aventis). Dr Hankey has served on an Atherothrombosis Advisory Board for, and received honoraria from, Sanofi-Aventis and Bristol-Myers Squibb. Dr Norman has served on an Advisory Board for, and received research funding from, Sanofi-Aventis.

\section{References}

Adler AI, Stevens RJ, Neil A, et al. 2002. UKPDS 59: Hyperglycaemia and other potentially modifiable risk factors for peripheral vascular disease in type 2 diabetes. Diabetes Care, 25:894-9.

American Diabetes Association. 2003. Peripheral arterial disease in people with diabetes. Diabetes Care, 26:3333-41.

Antithrombotic Trialists' Collaboration. 2002. Collaborative meta-analysis of randomised trials of antiplatelet therapy for prevention of death, myocardial infarction, and stroke in high risk patients. $B M J, 324: 71-86$.

Aronow WS. 2004. Management of peripheral arterial disease of the lower extremities in elderly patients. J Gerontol, 59A:172-7.

Aronow WS. 2006. Drug treatment of peripheral arterial disease in the elderly. Drugs Aging, 23:1-12.

Beckman JA, Jaff MR, Creager MA. 2006. The United States Preventive Services Task Force Recommendation Statement on Screening for Peripheral Arterial Disease: More Harm Than Benefit? Circulation, 114:861-6.

Beebe HG, Dawson DL, Cutler BS, et al. 1999. A new pharmacological treatment for intermittent claudication: Results of a randomized, multicenter trial. Arch Int Med, 159:2041-50.

Blood pressure lowering treatment trialists' collaboration. 2003. Effects of different blood pressure-lowering regimeson major cardiovascular events: results of prospectively designed overviews of randomised trials. Lancet, 362:1527-35.

Bradbury AW. 2003. The role of cilostazol in the management of intermittent claudication. Int J Clin Pract, 57:405-9.

CAPRIE Steering Committee. 1996. A randomised, blinded, trial of clopidogrel versus aspirin in patients at risk of ischaemic events (CAPRIE). Lancet, 348:1329-39.

Cariski AT. 2001. Cilostazol: a novel treatment option in intermittent claudication. Int J Clin Pract, 119:11-18.

Dawson DL, Cutler BS, Hiatt WR, et al. 2000. A comparison of cilostazol and pentoxifylline for treating intermittent claudication. Am J Med, 109:523-30.

Dawson DL, Cutler BS, Meissner MH, et al. 1998. Cilostazol has beneficial effects in treatment of intermittent claudication: Results from a multicenter, randomized, prospective, double-blind trial. Circulation, 98:678-86.

Dawson DL. 2001. Comparitive effects of cilostazol and other therapies for intermittent claudication. Am J Cardiol, 87:19D-27D.

Dormandy JA, Rutherford RB. 2000. Management of peripheral arterial disease. J Vasc Surg, 31:S1-S296.

Elam MB, Heckman J, Crouse JR, et al. 1998. Effect of novel antiplatelet agent cilostazol on plasma lipoproteins in patients with intermittent claudication. Arterioscler Thromb Vasc Biol, 181:942-7.

Erez G, Leitersdorf E. 2007. The Rationale for Using HMG-CoA Reductase Inhibitors ('Statins') in Peripheral Arterial Disease. Eur J Vasc Endovasc Surg, 33:192-201.
Fowler B, Jamrozik K, Norman P, et al. 2002. Improving maximum walking distance in early peripheral arterial disease: randomised controlled trial. Austr J Physiother, 48:269-75.

Fowler B, Jamrozik K, Norman P, et al. 2002. Prevalence of peripheral arterial disease: persistence of excess risk in former smokers. Aust NZ $J$ Pub Health, 26:219-24.

Garg PK, Tian L, Criqui MH, et al. 2006. Physical activity during daily life and mortality in patients with peripheral arterial disease. Circulation, $114: 242-8$.

Girolami B, Bernardi E, Prins MH, et al. 1999. Treatment of intermittent claudication with physical training, smoking cessation, pentoxifylline or nafronyl: a meta-analysis. Arch Int Med, 159:337-45.

Hankey GJ, Norman PE, Eikelboom JW. 2006. Medical management of peripheral arterial disease. JAMA, 295:547-53.

Heart Outcome Prevention Evaluation Study Investigators. 2000. Effects of an angiotensin-converting-enzyme inhibitor, Ramapril, on cardiovascular events in high-risk patients. $N$ Engl J Med, 342:145-53.

Heart Protection Study Collaborative Group. 2002. MRC/BHF Heart Protection Study of cholesterol lowering with simvastatin in 20,536 high-risk individuals: a randomised placebo-controlled trial. Lancet, 360:7-22.

Hiatt W. 2001. Medical treatment of peripheral arterial disease and claudication. N Engl J Med, 344:1608-21.

Hiatt WR, Hoag S, Hamman RF. 1995. Effect of diagnostic criteria on the prevalence of peripheral arterial disease. Circulation, 91:1472-9.

Hobbs SD, Marshall T, Fegan C, et al. 2007. The effect of supervised exercise and cilostazol on coagulation and fibrinolysis in intermittent claudication: A randomised controlled trial. J Vasc Surg, 45:65-70.

Liu Y, Shakur Y, Yoshitake MM, et al. 2001. Cilostazol (Pletal): a dual inhibitor of cyclic nucleotide phosphdiesterase type 3 and adenosine uptake. Cardiovac Drug Rev, 19:369-86.

Matousovic K, Grande JP, Chini CCS, et al. 1995. Inhibitors of cyclic nucleotide phophdiesterase isoenzymes type-III and type-IV suppress mitogensis of rat mesangial cells. J Clin Invest, 96:401-10.

McDermott MM, Greenland P, Liu K, et al. 2001. Leg symptoms in peripheral arterial disease. Associated clinical characteristics and functional impairment. JAMA, 286:1599-606.

Mehler P, Coll J, Estacio R, et al. 2003. Intensive blood pressure control reduces the risk of cardiovascular events in patients with peripheral arterial disease and type 2 diabetes. Circulation, 107:753-6.

Money SR, Herd JA, Isaacsohn JL, et al. 1998. Effect of cilostazol on walking distances in patients with intermittent claudication caused by peripheral vascular disease. J Vasc Surg, 27:267-75.

Motoyashiki T, Morita T, Ueki H. 1996. Involvement of the rapid increase in cAMP content in the vandate-stimulated release of lipoprotein lipase activity from rat fat pads. Biol Pharmacol Bull, 19:1412-16.

Muntner P, Wildman RP, Reynolds K, et al. 2005. Relationship between HbAic level and peripheral arterial disease. Diabetes Care, 28:1891-7.

Naylor A, Webb J, Fowkes F, et al. 1988. Trends in abdominal aortic aneurysm surgery in Scotland (1971-1984). Eur J Vasc Surg, 2:217-21.

Newman AB, Shemanski L, Manolio TA, et al. 1999. Ankle-arm index as a predictor of cardiovascular disease and mortality in the Cardiovascular Health Study. Arterioscler Thromb Vasc Biol, 19:538-45.

Norgren L, Hiatt WR, Dormandy JA, et al. 2007. Inter-Society Consensus for the Management of Peripheral Arterial Disease (TASC II). Eur J Vasc Endovasc Surg, 33:S1-S75.

Otsuka. Otsuka 21-94-201. 1996b. A randomised double blind study of the effect of cilostazol versus placebo on walking distances in patients with intermittent claudication secondary to peripheral vascular disease. Internal report. Unpublished data.: Otsuka Pharmaceuticals.

Otsuka. Otsuka 21-95-201. 1996a. A randomised double-blind study of the safety and efficacy of two different doses of cilostazol versus placebo in patients with intermittent claudication secondary to peripheral vascular disease. Internal Report. Unpublished data.: Ostuka Pharmaceuticals.

Pedersen TR, Kjekshus J, Pyorala K, et al. 1998. Effect of simvastatin on ischemic signs and symptoms in the Scandanavian Simvastatin Survival Study (4S). Am J Cardiol, 81:333-5. 
Pratt CM. 2001. Analysis of the cilostazol safety database. Am J Cardiol, 87:28D-33D.

Regensteiner JG, Ware JE, McCarthy WJ, et al. 2002. Effect of cilostazol on treadmill walking, community-based walking ability, and healthrelated qualitiy of life in patients with intermittent claudication due to peripheral arterial disease: Metanalysis of six randomized control trials. J Am Geriatric Soc, 50:1939-46.

Reilly MP, Mohler ER. 2001. Cilostazol: Treatment of intermittent claudication. Ann Pharmacother, 35:48-56.

Rendell M, Cariski AT, Hittel N, et al. 2002. Ciolstazol treatment of claudication in diabetic patients. Curr Med Res Opin, 18:479-87.

Selvin E, Erlinger T. 2004. Prevalence of and risk factors for peripheral arterial disease in the United States. Results from the National Health and Nutrition Examination Survey 1999-2000. Circulation, 110:738-743.

Shephard R, Balady G. 1999. Exercise as cardiovascular therapy. Circulation, 99:963-72.

Strandness DE, Dalman RL, Panian S, et al. 2002. Effect of cilostazol in patients with intermittent claudication: A randomized, double-blind, placebo-controlled study. Vasc Endovasc Surg, 36:83-91.
Takahashi S, Oida K, Fujiwara R, et al. 1992. Effect of cilostazol, a cyclic AMP phosphodiesterase inhibitor, on the proliferation of rat aortic smooth muscle in culture. J Cardiovasc Pharmacol, 20:900-6.

Thompson PD, Zimet R, Forbes WP, et al. 2002. Meta-analysis of results from eight randomized, placebo-controlled trials on the effect of cilostazol on patients with intermittent claudication. Am J Cardiol, 90:1314-19.

Willgendael EM, Teijink JAW, Bartelink M-L, et al. 2004. Influence of smoking on incidence and prevalence of peripheral arterial disease. J Vasc Surg, 40:1158-65.

Wind J, Koelemay MJW. 2007. Exercise therapy and the additional effect of supervision on exercise therapy in patients with intermittent claudication. Systematic review of randomised controlled trials. Eur J Vasc Endovasc Surg, 34:19.

Yusef S, Hawken S, Ounpuu S, et al. 2005. Obesity and the risk of myocardial infarction in 27,000 participants from 52 countries: a case-control study. Lancet, 366:1640-9. 
